\title{
RATIONAL ELIMINATION ALGORITHM AND APPLICATIONS
}

\author{
BÉchir DAli AND MOHAMEd ElghaOUI
}

Abstract. Given a matrix $A \in \mathbb{R}^{m \times n}$, we develop an algorithm called the rational elimination algorithm which ressembles the algorithm elimination except that the pivot (the leading coefficient) is a sequence of real independent numbers over $\mathbb{Q}$. This algorithm is used to calculate the rank of $A$ over $\mathbb{Q}$ and to seek rational solutions to any linear system $A x=b$ with $b \in \mathbb{R}^{m}$. We also present a criterion for testing the density of additive subgroups of $\mathbb{R}^{n}$ which needs the rank of a certain matrix over $\mathbb{Q}$. Finally, we apply such algorithm for testing the regularity of an orbit under the linear continuous action of some subgroup of $G L(V)$ where $V$ is finite dimensional real vector space.

Mathematics subject classification (2020): 15A06, 15A24, 15B36, 11 J72.

Keywords and phrases: Irrationality, linear dependence over a field, rational numbers, rational elimination algorithm.

\section{REFERENCES}

[1] A. Ayadi And H. Marzougui, Dense orbits for abelian subgroups of $G L(n, \mathbb{C})$, Foliations 2005 : World Scientific, Hackensack, NJ, (2006), 47-69.

[2] D. Arnal, B. Dali, B. Currey, V. Oussa, Regularity of abelian linear actions, Commutative and noncommutative harmonic analysis and applications, 89-109, Contemp. Math., 603, Amer. Math. Soc., Providence, RI, 2013.

[3] Didier Arnal, Bradley Currey, Vignon Oussa, Characterization of regularity for a connected Abelian action, Monatsh. Math. Volume 180. Issue 1, pp. 1-37, doi:10.1007/s00605-015-0811$\mathrm{y}$.

[4] Elghaoui, Mohamed, And Adlene Ayadi, Rational Criterion Testing the Density of Additive Subgroups of $\mathbb{R}^{n}$ and $\mathbb{C}^{n}$, Applied General Topology, vol. 16, no. 2, Oct. 2015, pp. 127-139, doi:10.4995/agt.2015.3257.

[5] Mohamed Elghaoui And Adlene AyAdi, Algorithm testing for the hypercyclicity of finitely abelian subgroups of $G L(n, \mathbb{C})$, Operators and Matrices Volume 10, Number 3 (2016), 669-678, doi:10.7153/oam-10-40.

[6] O. L. Mangasarian, And M. C. FERris, Uniqueness of Integer Solution of Linear Equations, Optimization Letters, vol. 4, no. 4, Nov. 2010, pp. 559-65, doi:10.1007/s11590-010-0183-0.

[7] M. Waldschmidt, Topologie des points rationnels, Cours de troisième Cycle, Université P. et M. Curie (Paris VI), (1994/95).

[8] Martin, RichaRd KiPP, Large Scale Linear and Integer Optimization: A Unified Approach, Springer US, 1999, doi:10.1007/978-1-4615-4975-8. 